\title{
Pre-radiotherapy lymphocyte count and platelet-to-lymphocyte ratio may improve survival prediction beyond clinical factors in limited stage small cell lung cancer: model development and validation
}

\author{
Yishan Yu ${ }^{1,2}$, Linlin Wang ${ }^{2 \#}$, Shufen $\mathrm{Cao}^{3}$, Siming Gao ${ }^{1,2}$, Weili Wang ${ }^{4}$, Lianne Mulvihill ${ }^{4}$, Mitchell Machtay ${ }^{4}$, \\ Pingfu $\mathrm{Fu}^{3}$, Jinming $\mathrm{Yu}^{2 \#}$, Feng-Ming (Spring) Kong ${ }^{4,5 \#}$ \\ ${ }^{1}$ School of Medicine, Shandong University, Jinan, China; ${ }^{2}$ Department of Radiation Oncology, Shandong Cancer Hospital and Institute, Shandong \\ First Medical University and Shandong Academy of Medical Sciences, Jinan, China; ${ }^{3}$ Department of Population and Quantitative Health Sciences, \\ Case Western Reserve University School of Medicine, Cleveland, OH, USA; ${ }^{4}$ Department of Radiation Oncology, Seidman Cancer Center, Case \\ Western Reserve University School of Medicine, University Hospitals Cleveland Medical Center, Cleveland, OH, USA; ${ }^{5}$ Department of Clinical \\ Oncology, Hong Kong University Shenzhen Hospital, Li Ka Shing Medical School, The University of Hong Kong, Shenzhen, China \\ Contributions: (I) Conception and design: L Wang, J Yu, FM Kong; (II) Administrative support: M Machtay, J Yu, FM Kong; (III) Provision of study \\ materials or patients: L Wang, J Yu; (IV) Collection and assembly of data: Y Yu, L Wang, S Gao; (V) Data analysis and interpretation: Y Yu, P Fu, S \\ Cao, W Wang, FM Kong; (VI) Manuscript writing: All authors; (VII) Final approval of manuscript: All authors. \\ \#These authors contributed equally to this work. \\ Correspondence to: Jinming Yu, MD, PhD. Department of Radiation Oncology, Shandong Cancer Hospital and Institute, Shandong First Medical \\ University and Shandong Academy of Medical Sciences, Jinan, China. Email: sdyujinming@126.com; Linlin Wang, MD, PhD. Department of \\ Radiation Oncology, Shandong Cancer Hospital and Institute, Shandong First Medical University and Shandong Academy of Medical Sciences, Jinan, \\ China. Email: wanglinlinatjn@163.com; Feng-Ming (Spring) Kong, MD, PhD, FACR, FAAWR, FASTRO. Department of Clinical Oncology, Hong \\ Kong University Shenzhen Hospital, Li Ka Shing Medical School, The University of Hong Kong, Shenzhen, China. Email: kong0001@hku.hk.
}

Background: Few small sample size studies have reported lymphocyte count was prognostic for survival in small-cell lung cancer (SCLC). This study aimed to validate this finding, to build prediction model for overall survival (OS) and to study whether novel models that combine lymphocyte-related variables can predict OS more accurately than a conventional model using clinical factors alone in a large cohort of limited-stage SCLC patients.

Methods: This study enrolled 544 limited-stage SCLC patients receiving definitive chemo-radiation with pre-radiotherapy lymphocyte-related variables including absolute lymphocyte count (ALC), platelet-tolymphocyte ratio (P/L ratio), neutrophil-to-lymphocyte ratio (N/L ratio), and lymphocyte-to-monocyte ratio (L/M ratio). The primary endpoint was OS. These patients were randomly divided into a training dataset $(n=274)$ and a validation dataset $(n=270)$. Multivariate survival models were built in the training dataset, and the performance of these models were further tested in the validation dataset using the concordance index (C-index).

Results: The median follow-up time was 36 months for all patients. In the training dataset, univariate analysis showed that $\mathrm{ALC}(\mathrm{P}=0.020)$ and $\mathrm{P} / \mathrm{L}$ ratio $(\mathrm{P}=0.023)$ were significantly correlated with $\mathrm{OS}$, while $\mathrm{L} / \mathrm{M}$ ratio $(\mathrm{P}=0.091)$ and $\mathrm{N} / \mathrm{L}$ ratio $(\mathrm{P}=0.436)$ were not. Multivariate modeling demonstrated the significance of $\mathrm{ALC}(\mathrm{P}=0.063)$ and $\mathrm{P} / \mathrm{L}$ ratio $(\mathrm{P}=0.003)$, and the improvement for $\mathrm{OS}$ prediction in combined models with the addition of ALC $(\mathrm{C}$-index $=0.693)$ or $\mathrm{P} / \mathrm{L}$ ratio $(\mathrm{C}$-index $=0.688)$ over the conventional model $(\mathrm{C}$-index $=0.679)$. The validation dataset analysis confirmed a modest improvement of $\mathrm{C}$-index with the addition of ALC or P/L ratio. All these models showed reasonable discriminations and calibrations.

Conclusions: This study validated the significant value of pre-radiotherapy ALC and P/L ratio on OS in limited-stage SCLC. The combined model with ALC or P/L ratio showed additional OS prediction values than the conventional model with clinical factors alone. 


\begin{abstract}
Keywords: Limited-stage small-cell lung cancer (SCLC); overall survival (OS); predictive model; lymphocyte count; platelet-to-lymphocyte ratio
\end{abstract}

Submitted May 13, 2020. Accepted for publication Jun 09, 2020.

doi: $10.21037 /$ tlcr-20-666

View this article at: http://dx.doi.org/10.21037/tlcr-20-666

\section{Introduction}

Small-cell lung cancer (SCLC) accounts for approximately $15 \%$ of all lung cancers and is characterized by rapid tumor growth, aggressive progression, and early metastatic dissemination (1). Only one-third of patients present with limited-stage SCLC at diagnosis, which is initially chemoresponsive. However, due to its aggressive nature, limitedstage SCLC is typified by rapid recurrence and extensive metastatic dissemination, resulting in death at a median of 15 to 20 months (2).

For limited-stage SCLC patients, definitive chemoradiation is the standard therapy. Prognostic markers have been extensively investigated for SCLC patients receiving standard therapy. These clinical markers include patient factors such as age, gender, performance status, tobacco exposure, and body mass index (BMI), tumor factors such as tumor stage, and therapeutic factors such as the timing of thoracic radiotherapy, concurrent chemotherapy or not, the cycles of chemotherapy, and the receipt of prophylactic cranial irradiation (PCI) (3-7).

Immune and inflammation are critical for tumor progression, metastatic dissemination, and treatment resistance (8). Absolute lymphocyte count (ALC), plateletto-lymphocyte ratio (P/L ratio), neutrophil-to-lymphocyte ratio (N/L ratio), and lymphocyte-to-monocyte ratio (L/M ratio) were related to survival outcomes in various cancers (9-11). However, the prognostic value of the L/M ratio has not been explored in SCLC and only a few studies involving small sample sizes have reported the prognostic significance of ALC, N/L ratio, and P/L ratio in SCLC with inconsistent results $(6,12-14)$. It is also important to note that no effort has been made to incorporate these hematological factors along with clinical factors into the survival model for clinical applications of SCLC.

In this study, we hypothesized that lymphocyte-related variables including $\mathrm{ALC}, \mathrm{P} / \mathrm{L}$ ratio, N/L ratio, and $\mathrm{L} / \mathrm{M}$ ratio at pre-radiotherapy (pre-RT) are significant for survival, and that the addition of these variables can improve the accuracy for survival prediction in SCLC.
To verify this hypothesis, we developed and validated the combined survival models based on significant lymphocyterelated variables and clinical factors. We then compared the performances of these combined models to the conventional model built from significant clinical factors alone using the concordance index (C-index) as well as the time-dependent receiver operating characteristic (ROC) analysis, with the area under the curves as the model performance metric.

We present the following article in accordance with the STROBE reporting checklist (available at http://dx.doi. org/10.21037/tlcr-20-666).

\section{Methods}

\section{Study population}

From 2012 to 2017, 544 patients with limited-stage SCLC were included from Shandong Cancer Hospital (Figure S1). The institutional review board of this hospital approved this retrospective study. The study was conducted in accordance with the Declaration of Helsinki (as revised in 2013). Because of the retrospective nature of the research, the requirement for informed consent was waived. Inclusion criteria included (I) at least 18 years old; (II) pathologically or cytologically confirmed SCLC; (III) clinically staged limited disease; (IV) treatment with definitive chemoradiation. Patients were excluded if (I) complete-bloodcount (CBC) data was not available within one week before thoracic radiotherapy; (II) detailed information of radiotherapy was not available; (III) patients had active infections or received corticoid before CBC; (IV) patients with concurrent or a history of other malignancies.

\section{Treatments and follow-up}

All patients received 3-dimensional conformal radiation therapy (3DCRT) or intensity modulated radiation therapy (IMRT). The radiation dose was generally given as 50-60 Gy at 2 Gy per fraction in once daily fractionation radiotherapy or 45 Gy at 1.5 Gy per fraction in twice daily 
treatment. The lungs and heart were contoured according to RTOG lung atlas for organs at risk (OARs) and the total body was created by an external contour of the body on each patient's CT simulation scan normally from midneck to mid-abdomen. The mean heart, lung, and body doses were then calculated. Platinum and etoposide were used as the standard chemotherapy regimens along with other platinum based regimens including irinotecan or taxol combined with platinum.

Patients were treated per standard of care of our hospital. After definitive therapy, patients were generally followed at regular intervals of every 3 months during the first year, every 6 months during the second year, and annually thereafter.

\section{Data collection}

Patient, tumor, and therapeutic characteristics were extracted from electronic medical records as detailed in Table 1. Patient-specific variables included age, gender, smoking history, Karnofsky Performance Status, and BMI. Tumor-specific variables included TNM stage (AJCC TNM staging system, $8^{\text {th }}$ edition, 2016). Treatment-specific variables included RT fractionation (once or twice daily), RT technique (3DCRT versus IMRT), chemotherapy schedules (concurrent versus sequential), the regimen of chemotherapy, cycles of induction and total chemotherapy, and the receipt of PCI. The dosimetric variables of important OARs such as heart, lung, and total body were also collected. The pre-RT hematological parameters including ALC, absolute white blood cell count (WBC), absolute neutrophil count (neutrophil), absolute monocyte count (monocyte), absolute platelet count (platelet), $\mathrm{N} / \mathrm{L}$ ratio, $\mathrm{P} / \mathrm{L}$ ratio, and $\mathrm{L} / \mathrm{M}$ ratio, were collected retrospectively.

\section{Statistical analysis}

The endpoint was overall survival (OS), which was calculated from the date of diagnosis to the date of death due to any cause and censored at the date of the last followup for survivors. Survival probabilities were estimated with the Kaplan-Meier curve, and outcomes by clinical stage were compared using log-rank tests. Patients were randomly divided into a training dataset $(\mathrm{n}=274)$ and a validation dataset $(n=270)$ for model development and validation. The difference between training dataset and validation dataset were determined by the chi squared test for categorical variables and the Mann-Whitney $U$ test for continuous variables. All the pre-RT hematological parameters were treated as continuous variables to preserve more information. Initially, the univariate proportional hazard Cox analysis was used to assess significant clinical variables for OS. The variables with $\mathrm{P}$ values $<0.150$ in the univariate analysis were candidates for the multivariate modeling by backward elimination procedure. Because of the significant correlations between ALC, P/L ratio, N/L ratio, and L/M ratio, each of these factors was tested separately in different models with other clinical factors. During the backward elimination process the multivariate Cox analysis started with all candidate variables. This was followed by deletion of the variable with the largest $P$ value, whose loss gives the most statistically insignificant deterioration of the model fit. This process was repeated until all the variables in the model had a $\mathrm{P}$ value of $<0.100$. Nomograms were generated through $\mathrm{R}$ software to predict survival rates at specific time points (3-year survival). The prediction performance of the models was evaluated by discrimination and calibration. The C-index was used to compare discrimination to predict the total survival of the different models. The time-dependent area under receiver operating characteristics (AUC) analysis was used to assess the different nomograms. The calibration plot, which visualizes the agreement between the observed and estimated survival probabilities, was generated. All tests were two-sided and $\mathrm{p}<0.05$ was considered statistically significant. Statistical analysis was performed using Stata/ MP 15.1 (Stata Corp LP, College Station, TX) and R (http://cran.r-project.org/) software.

\section{Results}

Of the 544 patients enrolled, the median age was 59 years with $69 \%$ males. Most (88\%) had stage III disease upon diagnosis. About $42 \%$ of patients (226 cases) received concurrent chemotherapy. Most (77\%) patients received more than 4 cycles of total chemotherapy, and 404 patients $(74 \%)$ were treated with once daily fractionation radiotherapy. After chemotherapy, 250 patients (46\%) received PCI. The mean of the total-body radiation dose was $7.3 \mathrm{~Gy}$. The mean ALC at pre-RT was $1.65 \times 10^{9}$ cells/L. Patient characteristics in the training dataset were comparable with those of the validation dataset (Table 1).

At the median follow-up time of 36.2 (95\% CI, 32.439.1) months, 238 patients were dead. The median survival 
Table 1 Comparison of patient characteristics between training and validation dataset

\begin{tabular}{|c|c|c|c|c|}
\hline Variables & All patients & Training dataset & Validation dataset & $P$ value* \\
\hline \multicolumn{5}{|l|}{ Patient and tumor factors } \\
\hline Age, median [range], years & $59[18-86]$ & $59[18-86]$ & 59 [32-81] & 0.203 \\
\hline \multicolumn{5}{|l|}{ Gender, n [\%] } \\
\hline Male & $376[69]$ & $187[68]$ & $189[70]$ & \\
\hline \multicolumn{5}{|l|}{ Smoking history, $\mathrm{n}[\%]$} \\
\hline No & $233[43]$ & $128[47]$ & $105[39]$ & 0.065 \\
\hline Yes & $311[57]$ & $146[53]$ & $165[61]$ & \\
\hline$\geq 90$ & $267[49]$ & $139[51]$ & $128[47]$ & \\
\hline \multicolumn{5}{|l|}{ BMI, n [\%] } \\
\hline$<18.5$ & 9 [2] & 5 [2] & 4 [2] & 0.124 \\
\hline 18.5 to $<25$ & $275[51]$ & $147[54]$ & $128[47]$ & \\
\hline 25 to $<30$ & $230[42]$ & $110[40]$ & $120[44]$ & \\
\hline$>30$ & $30[5]$ & $12[4]$ & $18[7]$ & \\
\hline \multicolumn{5}{|c|}{ Clinical stage [AJCC 8th], n [\%] } \\
\hline Stage I & 9 [2] & 5 [2] & 4 [2] & 0.811 \\
\hline \multicolumn{5}{|l|}{ Therapeutic factors } \\
\hline \multicolumn{5}{|l|}{ Chemotherapy schedule, $\mathrm{n}$ [\%] } \\
\hline Sequential & $318[58]$ & $171[62]$ & $147[54]$ & 0.059 \\
\hline Concurrent & 226 [42] & $103[38]$ & $123[46]$ & \\
\hline \multicolumn{5}{|c|}{ Regimen of chemotherapy, $\mathrm{n}$ [\%] } \\
\hline EP-based & $527[97]$ & $267[97]$ & $260[96]$ & 0.441 \\
\hline Others & $17[3]$ & 7 [3] & $10[4]$ & \\
\hline \multicolumn{5}{|c|}{ Cycles of induction chemotherapy, $\mathrm{n}[\%]$} \\
\hline$\leq 3$ & $328[60]$ & $160[58]$ & 168 [62] & 0.362 \\
\hline$>3$ & $216[40]$ & $114[42]$ & $102[38]$ & \\
\hline
\end{tabular}

Table 1 (continued) 
Table 1 (continued)

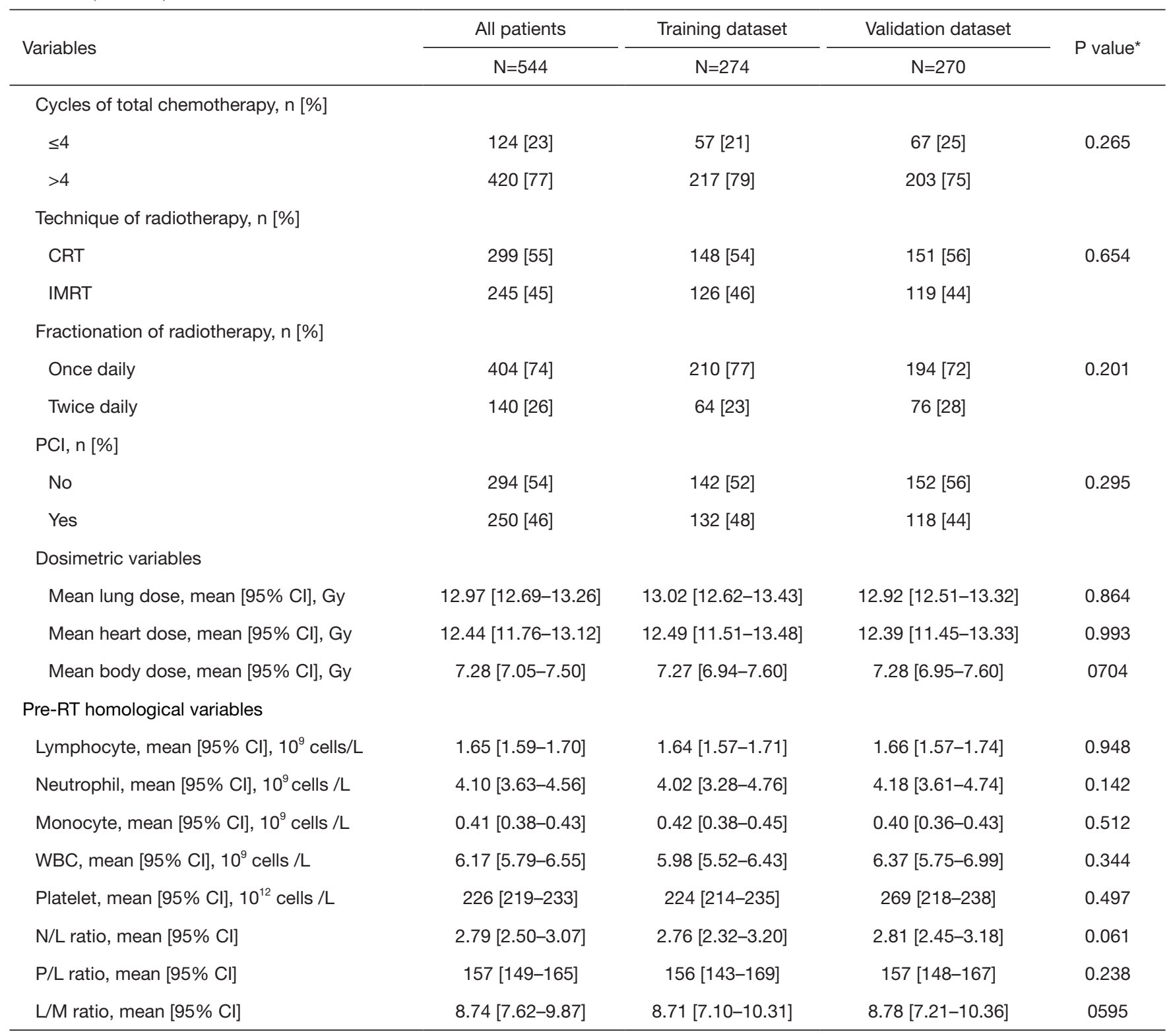

KPS, Karnofsky performance status; BMI, body mass index (calculated as weight in kilograms divided by height in meters squared); CRT, conformal radiation therapy; IMRT, intensity-modulated radiation therapy; PCI, prophylactic cranial irradiation; pre-RT, pre-radiotherapy; WBC, white blood cell; N/L ratio, neutrophil-to-lymphocyte ratio; P/L ratio, platelet-to-lymphocyte ratio; L/M ratio, lymphocyte-to-moncyte ratio; Stage IIIx: stage III with unknown stage IIIA, stage IIIB, stage IIIC. *, the P values were determined by the chi squared test for categorical variables and the Mann-Whitney $U$ test for continuous variables.

time was 36.1 (95\% CI, 32.4-39.1) months (40.1 months for training dataset and 35.9 months for validation dataset, logrank $\mathrm{P}=0.877$ ). The 3 -year survival rates of stage I, II, and III patients were $72.9 \%, 63.8 \%$, and $48.2 \%$, respectively. The median survival time of stage III patients was 34.6 months while those of stage I and II patients had not reached. In 428 of 478 stage III patients with IIIA, IIIB, or IIIC clearly classified, the median survival times were 36.1, 33.5, and 22.9 months, respectively (Figure 1).

In the training dataset, univariate analysis showed that age, clinical stage, chemotherapy schedule, cycles of induction chemotherapy, cycles of total chemotherapy, 

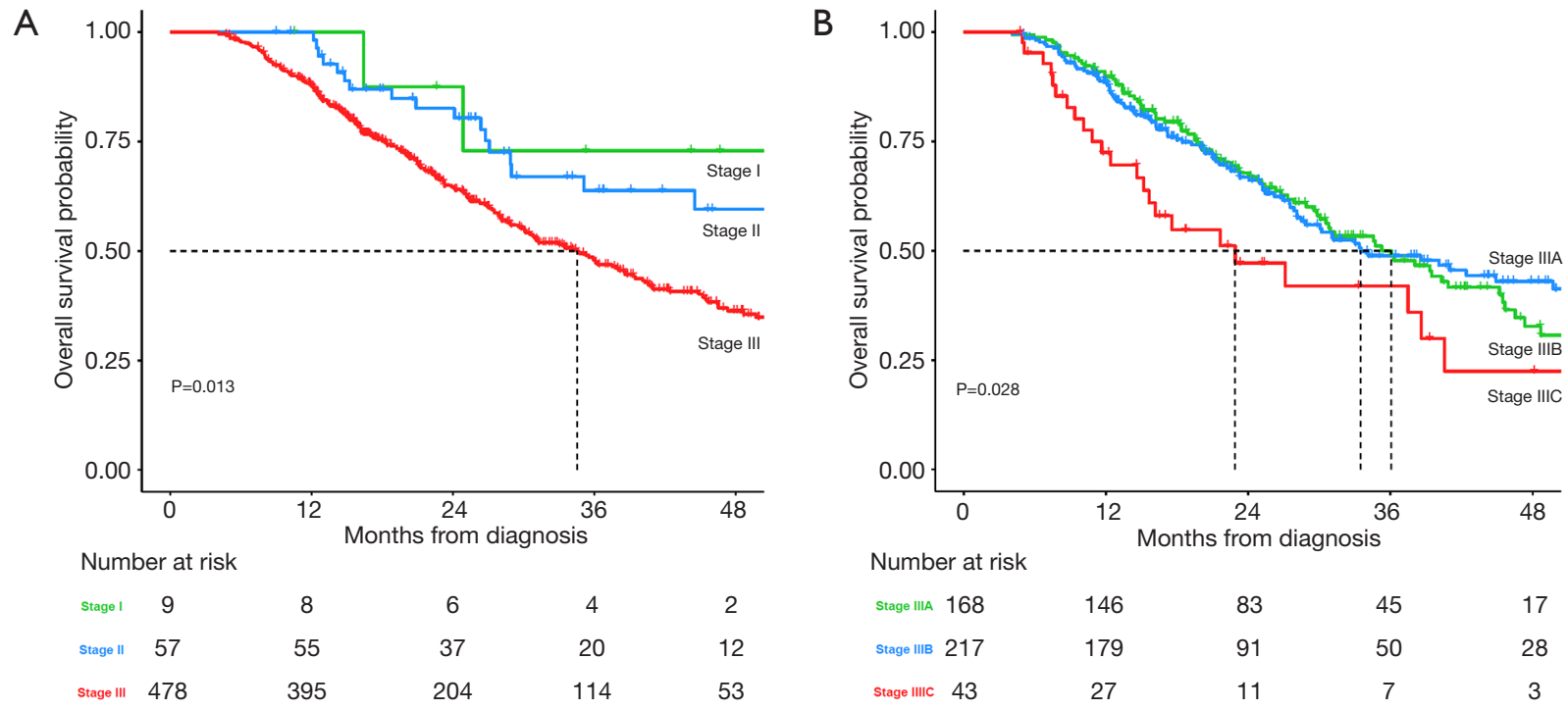

Figure 1 Overall survival stratified by clinical stage (AJCC TNM staging system 8th edition, 2016).

and mean body dose were significantly associated with OS (all $\mathrm{P}$ values $<0.05$ ) (Table 2). Pre-RT ALC $(\mathrm{HR}=0.679$ per $1 \times 10^{9}$ cells $/ \mathrm{L}$ increased, 95\% CI, 0.489-0.942, $\left.\mathrm{P}=0.020\right)$ and pre-RT P/L ratio (HR $=1.001$ per 1 unit increased, 95\% CI, $1.000-1.003, \mathrm{P}=0.023$ ) were also significantly associated with OS, while pre-RT N/L ratio (HR $=1.019$ per 1 unit, 95\% CI, 0.973-1.067, $\mathrm{P}=0.436$ ) and pre-RT L/M ratio (HR $=0.983$ per 1 unit, $95 \%$ CI, $0.964-1.003, \mathrm{P}=0.091$ ) were not.

For conventional model building, clinical variables with $\mathrm{P}$ values of $<0.150$ in the univariate analysis including age, gender, smoking history, BMI, clinical stage, chemotherapy schedule, cycles of induction chemotherapy, cycles of total chemotherapy, PCI, and mean body dose were selected as candidate variables. Finally, five variables including gender, clinical stage, cycles of induction chemotherapy, cycles of total chemotherapy, and PCI (all P values $<0.100$ ) were selected into the conventional survival model by the backward elimination procedure (Table 2).

During the combined model development process, ALC, $\mathrm{P} / \mathrm{L}$ ratio, and $\mathrm{L} / \mathrm{M}$ ratio (all $\mathrm{P}$ values $<0.150$ ) met the entry criteria and were selected as candidate variables for further modeling and could separately enter into multivariate modeling together with the above mentioned ten clinical variables. $\mathrm{L} / \mathrm{M}$ ratio failed during the backward elimination procedure due to the fact that it exceeded the predefined criteria for a $\mathrm{P}$ value of $<0.100$. Finally, two combined models, the ALC survival model and the $\mathrm{P} / \mathrm{L}$ ratio survival model, were developed. The $\mathrm{P} / \mathrm{L}$ ratio $(\mathrm{HR}=1.002$ per 1 unit, 95\% CI, 1.000-1.003, $\mathrm{P}=0.003$ ) remained significant while the ALC $\left(\mathrm{HR}=0.734\right.$ per $1 \times 10^{9}$ cells $/ \mathrm{L}, 95 \% \mathrm{CI}$, $0.529-1.017, \mathrm{P}=0.063$ ) was shown to be a marginally significant predictor for OS in the combined models (Table 2). Nomograms predicting 3-year survival are shown in Figure 2.

The model discrimination of survival models was evaluated by the $\mathrm{C}$-index. In the training dataset, the C-index of the conventional model was 0.679 (95\% CI, 0.626-0.731). With addition of lymphocyte-related variables, the C-indexes slightly increased to 0.693 (95\% CI, $0.643-0.743$, increased by $2.1 \%$ ) for the ALC survival model and 0.688 (95\% CI, 0.638-0.738, increased by $1.3 \%$ ) for the $\mathrm{P} / \mathrm{L}$ ratio survival model. The time-dependent AUCs of OS at 3 years were 0.686 (95\% CI, 0.604-0.767), 0.700 (95\% CI, 0.619-0.781), and 0.704 (95\% CI, $0.623-$ 0.784) for conventional model, ALC survival model, and $\mathrm{P} / \mathrm{L}$ ratio survival model, respectively (Figure $3 A$ ). In the validation dataset, the C-index was $0.656(95 \%$ CI, $0.605-0.707$ ), 0.662 (increased by $0.9 \%$ and $95 \%$ CI, $0.612-0.712$ ), and 0.659 (increased by $0.5 \%$ and $95 \% \mathrm{CI}$, 0.610-0.709) for the conventional model, ALC survival model, and P/L ratio survival model, respectively. The time-dependent AUCs of OS at 3-years of different models are shown in Figure $3 B$.

Most importantly, the calibration plots (Figure 4) showed that the predicted 3-year survival rate of validation dataset closely corresponded with the actual survival estimated by 
Table 2 Univariate analysis and model development for OS in training dataset

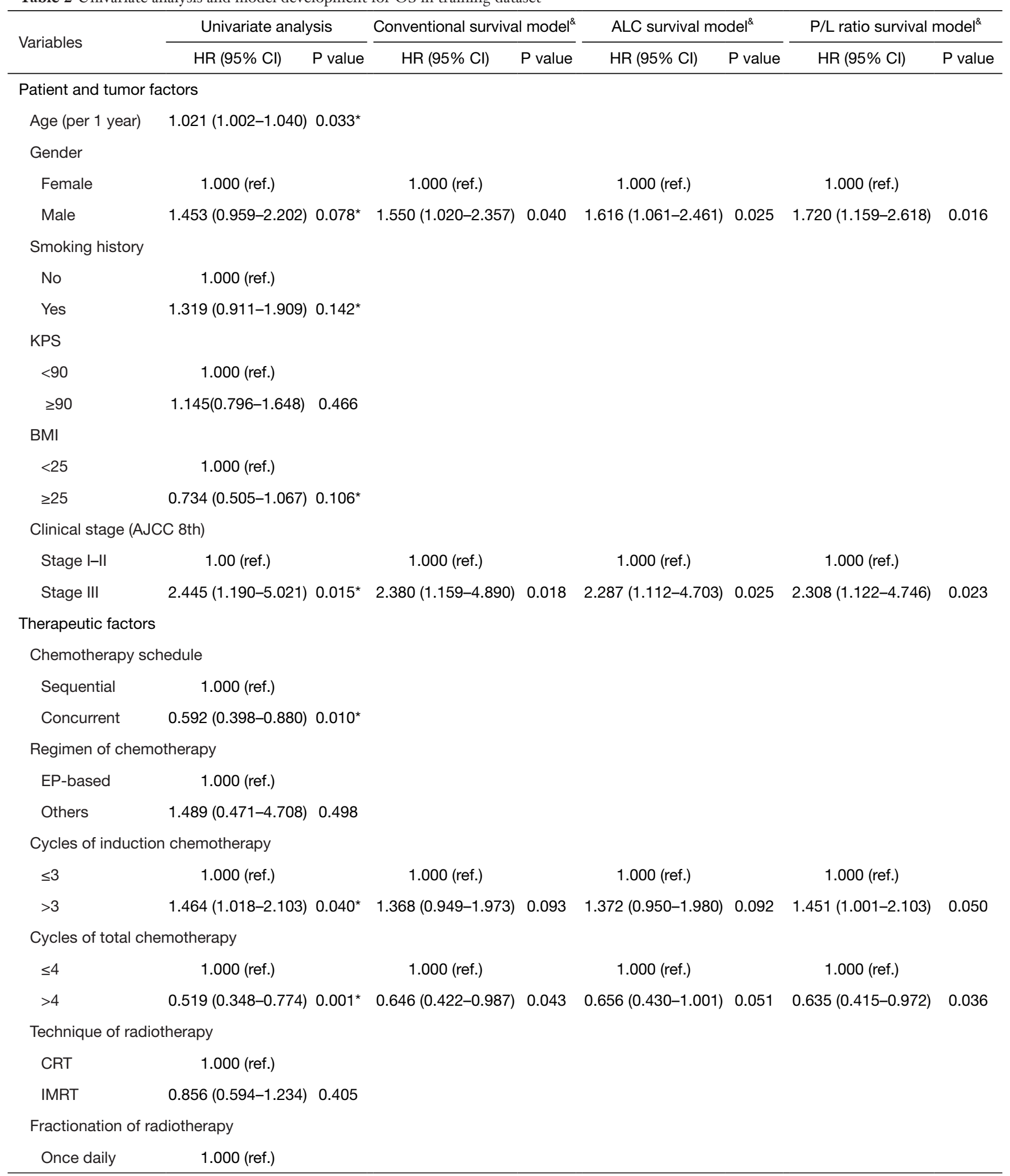

Table 2 (continued) 
Table 2 (continued)

\begin{tabular}{|c|c|c|c|c|c|c|c|c|}
\hline Variables & \multicolumn{2}{|c|}{ Univariate analysis } & \multicolumn{2}{|c|}{ Conventional survival model ${ }^{\&}$} & \multicolumn{2}{|c|}{ ALC survival model ${ }^{\&}$} & \multicolumn{2}{|c|}{$\mathrm{P} / \mathrm{L}$ ratio survival model ${ }^{\&}$} \\
\hline Twice daily & $1.321(0.872-2.000)$ & 0.189 & & & & & & \\
\hline \multicolumn{9}{|l|}{$\mathrm{PCl}$} \\
\hline No & 1.000 (ref.) & & 1.000 (ref.) & & 1.000 (ref.) & & 1.000 (ref.) & \\
\hline \multicolumn{9}{|l|}{ Dosimetric variables } \\
\hline $\begin{array}{l}\text { Mean lung dose, } \\
\text { (per } 1 \text { Gy) }\end{array}$ & $1.018(0.965-1.073)$ & 0.512 & & & & & & \\
\hline $\begin{array}{l}\text { Mean heart dose, } \\
\text { (per } 1 \text { Gy) }\end{array}$ & $1.004(0.981-1.026)$ & 0.757 & & & & & & \\
\hline $\begin{array}{l}\text { Lymphocyte, } \\
\text { (per } 1 \times 10^{9} \text { cells /L) }\end{array}$ & $0.679(0.489-0.942)$ & $0.020^{*}$ & & & $0.734(0.529-1.017)$ & 0.063 & & \\
\hline $\begin{array}{l}\text { Neutrophil, } \\
\left.\text { (per } 1 \times 10^{9} \text { cells } / L\right)\end{array}$ & $0.987(0.942-1.033)$ & 0.569 & & & & & & \\
\hline $\begin{array}{l}\text { Monocyte, } \\
\left.\text { (per } 1 \times 10^{9} \text { cells } / L\right)\end{array}$ & $1.108(0.76-1.816)$ & 0.684 & & & & & & \\
\hline $\begin{array}{l}\text { WBC, } \\
\left(\text { per } 1 \times 10^{9} \text { cells } / L\right)\end{array}$ & $0.996(0.949-1.045)$ & 0.860 & & & & & & \\
\hline $\begin{array}{l}\text { Platelet, } \\
\left.\text { (per } 1 \times 10^{12} \text { cells } / L\right)\end{array}$ & $0.999(0.997-1.001)$ & 0.474 & & & & & & \\
\hline
\end{tabular}

95\% Cl, 95\% confidence interval; HR, hazard ratio; pre-RT, pre-radiotherapy; KPS, Karnofsky performance status; BMI, body mass index (calculated as weight in kilograms divided by height in meters squared); CRT, conformal radiation therapy; IMRT, intensity-modulated radiation therapy; PCl, prophylactic cranial irradiation; WBC, white blood cell; N/L ratio, neutrophil-to-lymphocyte ratio; P/L ratio, platelet-to-lymphocyte ratio; L/M ratio, lymphocyte-to-monocyte ratio; ${ }^{*}$, the final multivariable Cox proportional hazard model was constructed by backward elimination; *, indicates the variables with $\mathrm{P}$ values $<0.15$ in the univariate analysis and these variables were chosen for the multivariate Cox regression model building process. 
A

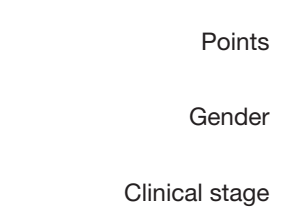

Cycles of induction ChT

Cycles of total ChT

$\mathrm{PCl}$

Total Points

Linear Predicto

3-year surviva

B

Pre-radiation Lymphocyte $\left(\times 10^{9}\right.$ cells/L)

Total Points

Linear Predicto

3-year survival

\section{C}

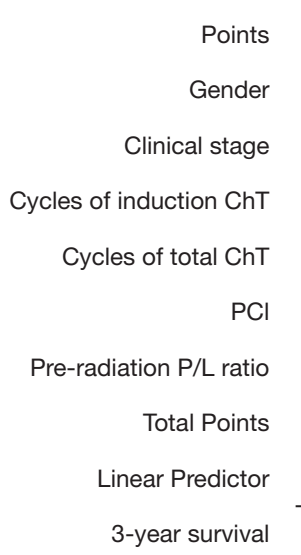

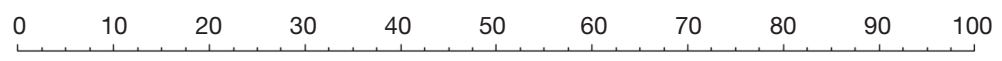

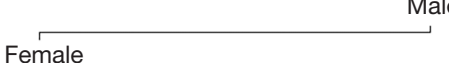

Stage III

Stage I-II

$>3$ cylcles

$\leq 3$ cylcles $\leq 4$ cylcles

$>4$ cylcles

No
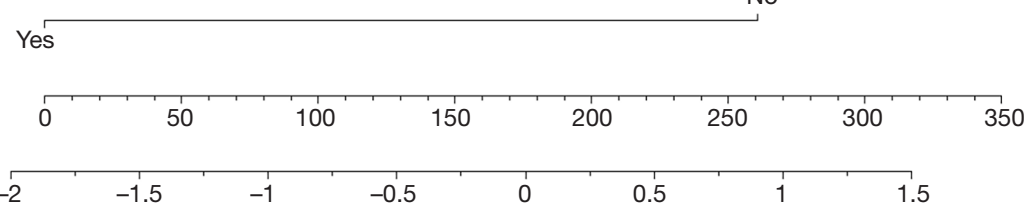

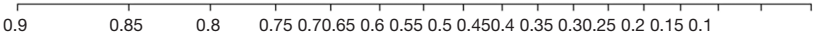

$0 \quad 10, \quad 20,30, \quad 40,50,60,70,80,90,100$

Male

Male
Female Stage III

Stage I-II >3 cylcles

$\leq 3$ cylcles $\quad \leq 4$ cylcles

$>4$ cylcles No

Yes

\begin{tabular}{lllllllllll}
\hline 5 & 4.5 & 4 & 3.5 & 3 & 2.5 & 2 & 1.5 & 1 & 0.5 & 0
\end{tabular}

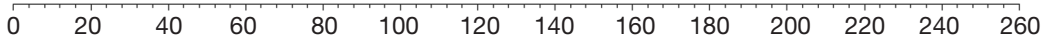

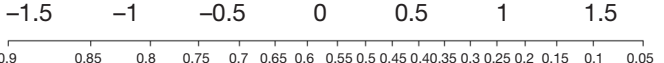

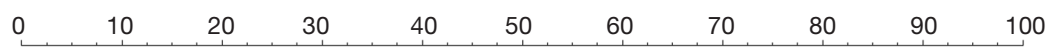

Female Male

Stage I-II $>3$ cylcles

$\leq 3$ cylcles $\leq 4$ cylcles

$>4$ cylcles No

Yes

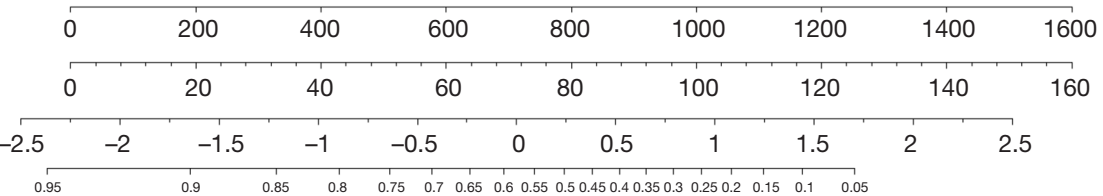

Figure 2 Nomogram for 3-year survival predicting in limited-stage SCLC. (A) Conventional nomogram with significant clinical factors; (B) pre-RT ALC survival nomogram with pre-RT ALC and significant clinical factors; (C) pre-RT P/L ratio survival nomogram with pre-RT $\mathrm{P} / \mathrm{L}$ ratio and significant clinical factors. SCLC, small cell lung cancer; ChT, chemotherapy; PCI, prophylactic cranial irradiation; pre-RT, pre-radiotherapy; ALC, absolute lymphocyte count; P/L ratio, platelet-to-lymphocyte ratio. 

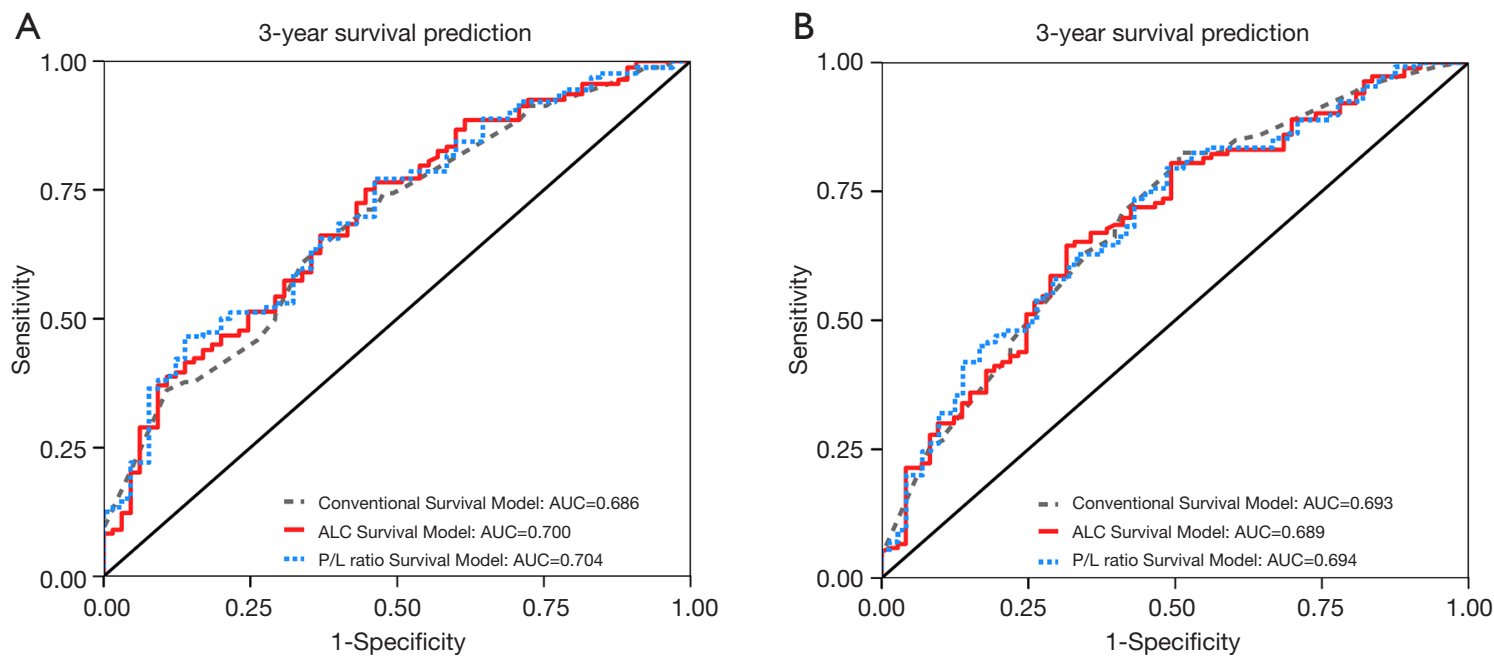

Figure 3 Time-dependent ROC curves by different models. 3-year OS prediction ROC curve in training dataset (A) and validation dataset (B). ROC, Receiver operative characteristic; OS, overall survival.
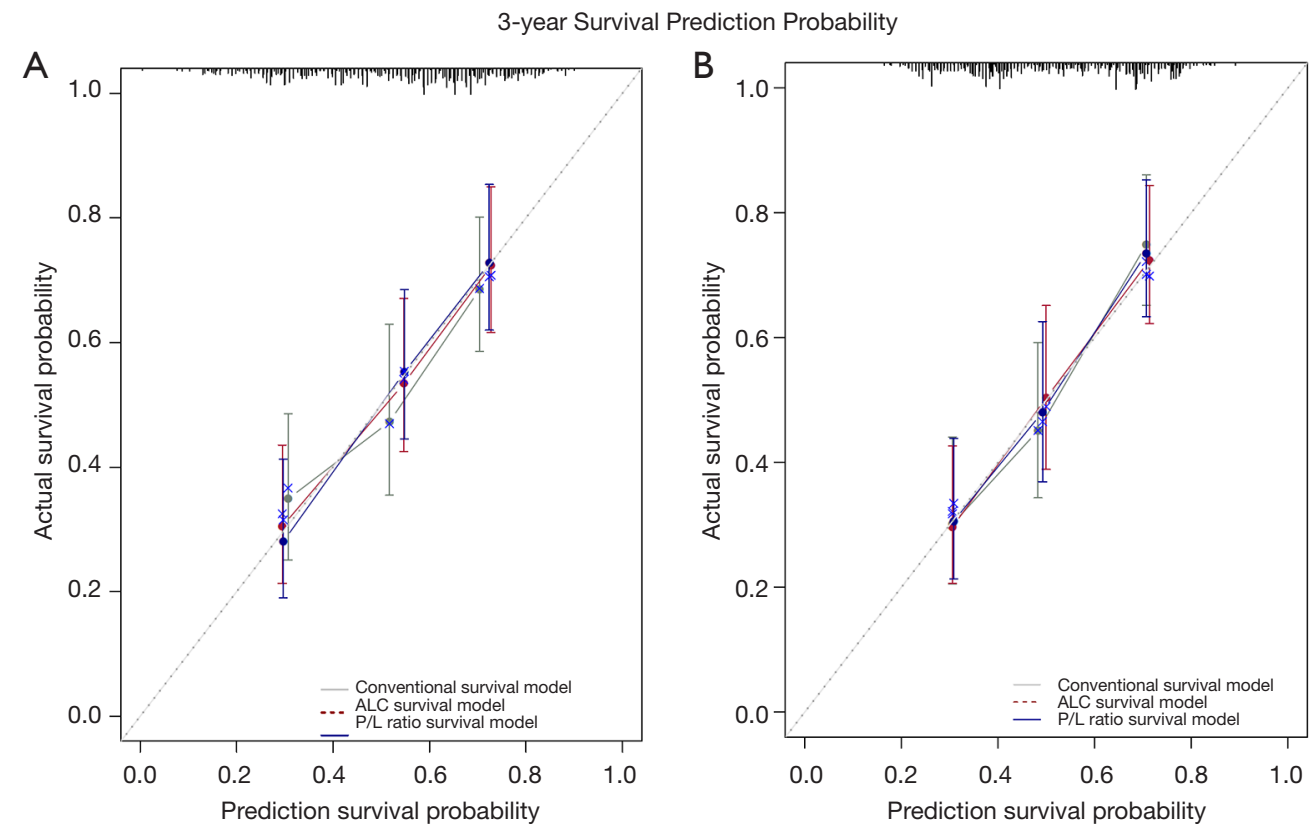

Figure 4 The calibration curves of nomograms for predicting 3-year overall survival in training dataset (A) and validation dataset (B), respectively. The axis is nomogram-predicted probability of survival and y-axis is actual survival. The reference line is $45^{\circ}$ and indicates almost perfect calibration.

the Kaplan-Meier method, which is represented by the dotted lines in both the training and validation datasets.

\section{Discussion}

In this study of 544 patients, we (I) validated that pre-
RT ALC and P/L ratio were significantly associated with OS while pre-RT N/L ratio and $\mathrm{L} / \mathrm{M}$ ratio were not, (II) demonstrated that the addition of ALC or P/L ratio into the conventional model including significant clinical factors showed a modest improvement of C-index in OS prediction in limited-stage SCLC patients, (III) generated 
OS predictive nomograms with reasonable discriminations and calibrations.

The findings of this large sample sized study can serve as an extended validation of the conventional blood testing during our daily practice. Differentials of blood cells, not being used in full extent in the clinic, could reflect an individual's immune and inflammation status, which are critical in tumor development and prognosis. Lymphocyte is known for its key role of host immune function, can be a reflection of host immunity. The significance of circulating ALC for OS is biologically sound and supported by the clinical literature (15-17) of various solid tumors. Several studies demonstrated the independent prognostic value of pre-treatment ALC, N/L ratio, $\mathrm{P} / \mathrm{L}$ ratio, and $\mathrm{L} / \mathrm{M}$ ratio in various cancers (9-11). Circulating neutrophils, a marker for bone marrow suppression after chemotherapy, are innate immune markers, which participate in the inflammatory process. The circulating neutrophil count is an indicator of systemic inflammation and is associated with poor survival in NSCLC (18). Interestingly, the percentage of circulating neutrophils is significantly higher in lung cancer patients than those in healthy patients (18). Platelets contribute to the immune modulating inflammatory process and cancer patients were reported to have increased platelet counts (10-57\%) (19). Importantly, platelets promote tumor development by acting as barriers for immune escape, resulting in abnormal vasculature and release of secretory factors such as thrombin and lysophosphatidic acid (20-22). Monocytes can regulate the immune response by a variety of chemokines. Tumor associated macrophages (TAM) derived from circulating monocytes could promote tumor progression, angiogenesis, and lymphangion genesis (23). Higher N/L and $\mathrm{P} / \mathrm{L}$ ratios indicate increased neutrophil or platelet counts and relatively decreased lymphocyte count, while higher L/M ratio indicates increased lymphocyte count and relatively decreased monocyte count. Thus, higher ALC and L/M ratio always indicate the protective systemic immunity and would be associated with better survival. In comparison, higher $\mathrm{N} / \mathrm{L}$ and $\mathrm{P} / \mathrm{L}$ ratios may be a reflection of impaired immunity or systematic inflammation and are associated with decreased survival.

Our study, may be the largest one reported thus far for ALC on survival in limited-stage SCLC, validated previous reports from other centers that ALC levels before treatment were significant for OS in patients with $\operatorname{SCLC}(6,24)$. Our result of pre-RT ALC as a significant marker for OS is consistent with report from others $(6,24)$, though chemotherapy before radiotherapy may change the lymphocyte count, which could also impact OS. In addition to validate ALC, our study also confirmed the survival significance of pre-RT ALC (marginally) and P/L ratio $(6,24,25)$, but failed to generate similar results on the significance of N/L ratio which was reported previously as significant factor in studies of small sample sizes $(6,24,26,27)$. Our result of pre-RT N/L ratio as a non-significant marker for OS is inconsistent with two other studies with 65 and 187 patients, indicating the significantly prognostic value of pretreatment N/L ratio for OS $(26,27)$. One reason to explain this difference may be the time-point in our study. The blood test was measured at pre-RT in our study while it was measured prior to any treatment in other studies. Another reason may be the different way of variable classifications, that is, we treated all variables as continuous variables while in almost all other studies, they treated the variables as categorical ones. Induction chemotherapy before radiotherapy may induce neutropenia, which could increase the risk of infection which may lead to death (28). Neutropenia is always linked with lower N/L ratios and inversely related to decreased survival. For the survival significance of the $\mathrm{P} / \mathrm{L}$ ratio, two studies in addition to this study have indicated the survival significance $(24,25)$, while two other studies have not $(26,27)$. A large cooperative study may be needed to confirm the significance of the ALC, P/L ratio, and N/L ratio. Our study is the first study to explore the association between $\mathrm{L} / \mathrm{M}$ ratio and $\mathrm{OS}$ in limited-stage SCLC, demonstrating that the $\mathrm{L} / \mathrm{M}$ ratio was not a significant predictor for OS. Further external validation is needed.

Our study is the first to use significant blood testing results to develop nomogram for survival prediction, by quantitatively computing the predictive values of each significant biomarker with combination of clinical significant factors. The nomogram seems to be a good way to present the effect of each significant variable in a prediction model. Each variable alone can only play a limited prognostic ability to assess the risk of death because of the complex nature and heterogeneous treatment choices of SCLC patients. Combining all of these independent factors together into one multivariate nomogram could improve the prediction ability, and multivariate nomograms provided more accurate predictions for the prognosis of SCLC patients than TNM stage and VALSG stage $(13,29)$. To better evaluate the prognostic effects of significant immune and inflammation biomarkers, specifically preRT ALC and P/L ratio in this study, we first developed two novel combined models, which featured pre-RT 
ALC or pre-RT P/L ratio. Compared to the conventional model of using clinically significant variables, these new models slightly improved the predictive accuracy of OS. Although improvement of C-index was numerically modest, especially in the specific-time nomogram, based on the effect size (hazard ratio) of pre-RT ALC or pre-RT P/L ratio, we believe it is valuable. Moreover, it also suggests the complexity of survival prediction and presence of other yet to be defined biomarkers that deserve the attentions of future studies.

This study is limited for its retrospective nature of the study from a single Institute, which carries all the weaknesses of such studies such as being unable to take all affecting factors into account. A study from a single institute may cause the selection bias of patients from the population. The apparently superior survival of our patients than most reported is likely due to the selection bias of better patients for clinical follow-up. However, the measurements of the $\mathrm{CBC}$ and differentials were objective and would not have made a difference in prospective analysis. One also have to note that the improvement of the lymphocyte-related variable models was numerically small, suggesting that more powerful biologic factors exist, all of which could be topics for future research. Indeed, findings from this study should be validated in larger prospective multicenter study.

In conclusion, this study validated the prognostic value of pre-RT ALC and P/L ratio, developed and validated models combining pre-RT ALC or P/L ratio with significant conventional clinical factors for OS prediction in limitedstage SCLC. The final prognostic models and nomograms with pre-RT ALC or P/L ratio showed reasonable discrimination and calibration and slightly improved the prediction value for OS compared to the conventional models. The applicability of pre-RT ALC or P/L ratio warrants further validation in prospective settings.

\section{Acknowledgments}

Funding: This work was supported in parts by the National Key Research and Development Program of China No. 2018YFC1313200 (PI: Yu), the National Cancer Institute, National Institutes of Health, R01 CA142840 (PI: Kong) and Shenzhen Science and Technology grant KQTD20180411185028798 (PI: Kong).

\section{Footnote}

Reporting Checklist: The authors have completed the
STROBE reporting checklist. Available at http://dx.doi. org/10.21037/tlcr-20-666

Data Sharing Statement: Available at http://dx.doi. org/10.21037/tlcr-20-666

Conflicts of Interest: All authors have completed the ICMJE uniform disclosure form (available at http://dx.doi. org/10.21037/tlcr-20-666). FMK serves as an unpaid editorial board member of Translational Lung Cancer Research from Apr 2016 to Jul 2021. The other authors have no conflicts of interest to declare.

Ethical Statement: The authors are accountable for all aspects of the work in ensuring that questions related to the accuracy or integrity of any part of the work are appropriately investigated and resolved. The study was conducted in accordance with the Declaration of Helsinki (as revised in 2013). The institutional review board of Shandong Cancer Hospital approved this retrospective study. Because of the retrospective nature of the research, the requirement for informed consent was waived.

Open Access Statement: This is an Open Access article distributed in accordance with the Creative Commons Attribution-NonCommercial-NoDerivs 4.0 International License (CC BY-NC-ND 4.0), which permits the noncommercial replication and distribution of the article with the strict proviso that no changes or edits are made and the original work is properly cited (including links to both the formal publication through the relevant DOI and the license). See: https://creativecommons.org/licenses/by-nc-nd/4.0/.

\section{References}

1. Gazdar AF, Bunn PA, Minna JD. Small-cell lung cancer: what we know, what we need to know and the path forward. Nat Rev Cancer 2017;17:725-37.

2. van Meerbeeck JP, Fennell DA, De Ruysscher DK. Smallcell lung cancer. Lancet 2011;378:1741-55.

3. Albain KS, Crowley JJ, LeBlanc M, et al. Determinants of improved outcome in small-cell lung cancer: an analysis of the 2,580-patient Southwest Oncology Group data base. J Clin Oncol 1990;8:1563-74.

4. Ou SH, Ziogas A, Zell JA. Prognostic factors for survival in extensive stage small cell lung cancer (ED-SCLC): the importance of smoking history, socioeconomic and marital statuses, and ethnicity. J Thorac Oncol 2009;4:37-43. 
5. Hasan S, White R, Renz P, et al. Optimal timing of thoracic radiotherapy in limited stage small cell lung cancer (SCLC) with daily fractionation: A brief report. Radiother Oncol 2019;132:23-6.

6. Suzuki R, Wei X, Allen PK, et al. Prognostic Significance of Total Lymphocyte Count, Neutrophil-to-lymphocyte Ratio, and Platelet-to-lymphocyte Ratio in Limited-stage Smallcell Lung Cancer. Clin Lung Cancer 2019;20:117-23.

7. Shepshelovich D, Xu W, Lu L, et al. Body Mass Index (BMI), BMI Change, and Overall Survival in Patients With SCLC and NSCLC: A Pooled Analysis of the International Lung Cancer Consortium. J Thorac Oncol 2019;14:1594-607.

8. Prasad N, Prasad R, Thornby J, et al. Lymphocyte replication in lung cancer patients undergoing radiotherapy. Oncology 1980;37:107-10.

9. Dupré A, Malik HZ. Inflammation and cancer: What a surgical oncologist should know. Eur J Surg Oncol 2018;44:566-70.

10. Yang S, Zhao K, Ding X, et al. Prognostic Significance of Hematological Markers for Patients with Nasopharyngeal Carcinoma: A Meta-analysis. J Cancer 2019;10:2568-77.

11. Venkatesulu BP, Mallick S, Lin SH, et al. A systematic review of the influence of radiation-induced lymphopenia on survival outcomes in solid tumors. Crit Rev Oncol Hematol 2018;123:42-51.

12. Cho O, Oh YT, Chun M, et al. Radiation-related lymphopenia as a new prognostic factor in limited-stage small cell lung cancer. Tumour Biol 2016;37:971-8.

13. Pan H, Shi X, Xiao D, et al. Nomogram prediction for the survival of the patients with small cell lung cancer. J Thorac Dis 2017;9:507-18.

14. Liu D, Huang Y, Li L, et al. High neutrophil-tolymphocyte ratios confer poor prognoses in patients with small cell lung cancer. BMC Cancer 2017;17:882.

15. Grossman SA, Ellsworth S, Campian J, et al. Survival in Patients With Severe Lymphopenia Following Treatment With Radiation and Chemotherapy for Newly Diagnosed Solid Tumors. J Natl Compr Canc Netw 2015;13:1225-31.

16. Tang C, Liao Z, Gomez D, et al. Lymphopenia association with gross tumor volume and lung V5 and its effects on non-small cell lung cancer patient outcomes. Int J Radiat Oncol Biol Phys 2014;89:1084-91.

17. Davuluri R, Jiang W, Fang P, et al. Lymphocyte Nadir and Esophageal Cancer Survival Outcomes After Chemoradiation Therapy. Int J Radiat Oncol Biol Phys 2017;99:128-35.

18. Barrera L, Montes-Servin E, Hernandez-Martinez JM, et al. CD47 overexpression is associated with decreased neutrophil apoptosis/phagocytosis and poor prognosis in non-small-cell lung cancer patients. Br J Cancer 2017;117:385-97.

19. Sierko E, Wojtukiewicz MZ. Platelets and angiogenesis in malignancy. Semin Thromb Hemost 2004;30:95-108.

20. Buergy D, Wenz F, Groden C, et al. Tumor-platelet interaction in solid tumors. Int J Cancer 2012;130:2747-60.

21. Ho-Tin-Noé B, Carbo C, Demers M, et al. Innate immune cells induce hemorrhage in tumors during thrombocytopenia. Am J Pathol 2009;175:1699-708.

22. Amo L, Tamayo-Orbegozo E, Maruri N, et al. Involvement of platelet-tumor cell interaction in immune evasion. Potential role of podocalyxin-like protein 1 . Front Oncol 2014;4:245.

23. Lin $\mathrm{Y}, \mathrm{Xu} \mathrm{J}$, Lan H. Tumor-associated macrophages in tumor metastasis: biological roles and clinical therapeutic applications. J Hematol Oncol 2019;12:76.

24. Suzuki R, Lin SH, Wei X, et al. Prognostic significance of pretreatment total lymphocyte count and neutrophil-tolymphocyte ratio in extensive-stage small-cell lung cancer. Radiother Oncol 2018;126:499-505.

25. Xie D, Marks R, Zhang M, et al. Nomograms Predict Overall Survival for Patients with Small-Cell Lung Cancer Incorporating Pretreatment Peripheral Blood Markers. J Thorac Oncol 2015;10:1213-20.

26. Kang MH, Go SI, Song HN, et al. The prognostic impact of the neutrophil-to-lymphocyte ratio in patients with small-cell lung cancer. Br J Cancer 2014;111:452-60.

27. Wang X, Teng F, Kong L, et al. Pretreatment neutrophilto-lymphocyte ratio as a survival predictor for small-cell lung cancer. Onco Targets Ther 2016;9:5761-70.

28. Pizzo PA. Management of fever in patients with cancer and treatment-induced neutropenia. N Engl J Med 1993;328:1323-32.

29. Wang S, Yang L, Ci B, et al. Development and Validation of a Nomogram Prognostic Model for SCLC Patients. J Thorac Oncol 2018;13:1338-48.

Cite this article as: $\mathrm{Yu} \mathrm{Y,} \mathrm{Wang} \mathrm{L}$, Cao S, Gao S, Wang W, Mulvihill L, Machtay M, Fu P, Yu J, Kong FM. Preradiotherapy lymphocyte count and platelet-to-lymphocyte ratio may improve survival prediction beyond clinical factors in limited stage small cell lung cancer: model development and validation. Transl Lung Cancer Res 2020;9(6):2315-2327. doi: $10.21037 /$ tlcr-20-666 


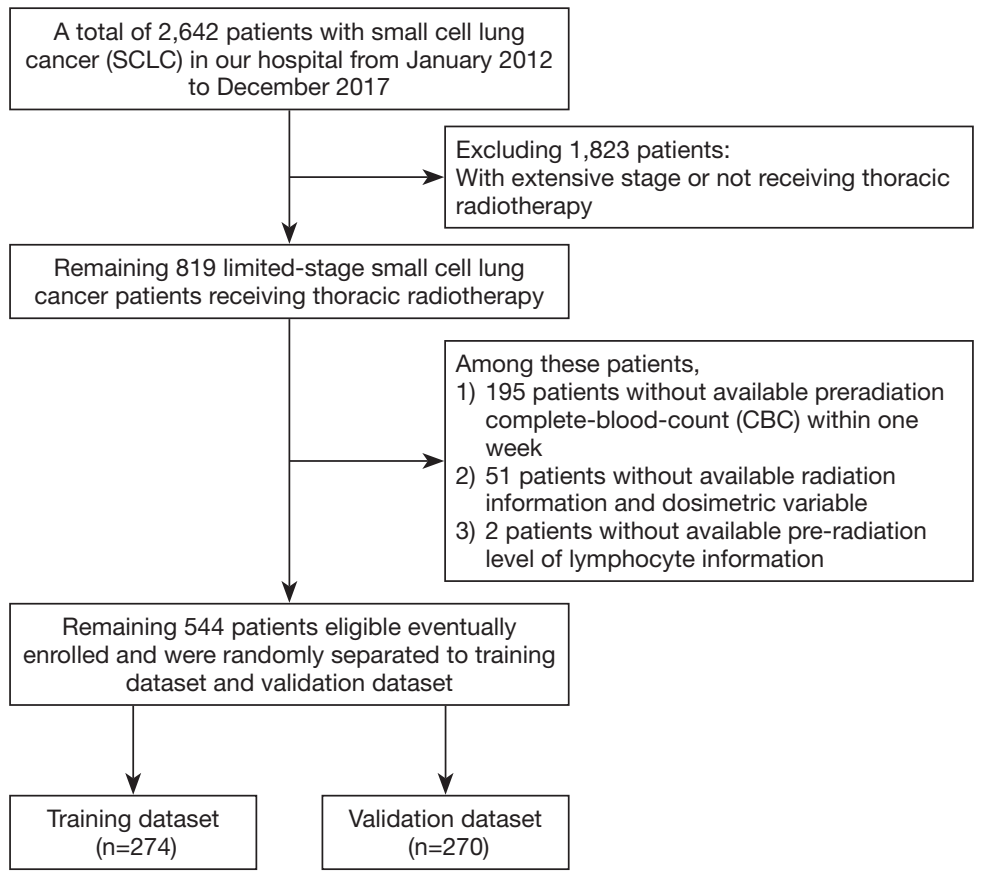

Figure S1 Flow diagram of study population. 